\title{
Questes
}

\section{Faire communauté : conclusion}

Julia Conesa Soriano et Julie Pilorget

\section{(2) OpenEdition}

Journals

Édition électronique

URL : http://journals.openedition.org/questes/4329

DOI : 10.4000/questes.4329

ISSN : 2109-9472

\section{Éditeur}

Les Amis de Questes

\section{Édition imprimée}

Date de publication : 6 mai 2016

Pagination : 157-160

ISSN : 2102-7188

\section{Référence électronique}

Julia Conesa Soriano et Julie Pilorget, «Faire communauté : conclusion», Questes [En ligne], 32 | 2016 mis en ligne le 10 mai 2016, consulté le 15 septembre 2020. URL : http://journals.openedition.org/ questes/4329 


\section{Conclusion}

\section{Julia Conesa Soriano et Julie PILORget}

Les articles réunis dans ces pages ont souligné l'importance de la notion de communauté pour la période médiévale, notamment à partir du Moyen Âge central. Ils reflètent surtout la diversité du fait communautaire médiéval, abordant tour à tour les communautés urbaines ${ }^{1}$, les communautés de métier ${ }^{2}$, la communauté chrétienne ${ }^{3}$ ou encore les communautés ethniques ${ }^{4}$. Ils apportent dans le même temps une réflexion sur la constitution de ces groupes et sur la position de l'individu par rapport à eux ${ }^{5}$, sur leurs modalités concrètes de coexistence ${ }^{6}$, autant que sur leur dissolution et les phénomènes d'exclusion qui accompagnent nécessairement leur existence ${ }^{7}$. À travers ces approches, se pose la question de savoir comment se forme et se maintient la communauté, comment on «fait communauté », quelle que soit la nature de cette dernière, dans le contexte médiéval.

Florian Besson ${ }^{8}$ passe par la notion de middle ground pour répondre à cette interrogation et ainsi définir le fait communautaire dans les États latins d'Orient. Plutôt que d'établir une distinction entre des groupes humains clairement délimités, la notion lui permet d'observer

\footnotetext{
${ }^{1}$ Voir, dans ce numéro, les articles de Cléo Rager, p. 123-138, et Diane Chamboduc de Saint-Pulgent, p. 103-122.

${ }^{2}$ Voir, dans ce numéro, l'article de Catherine Kikuchi, p. 139-156.

${ }^{3}$ Voir, dans ce numéro, l'article d' Élodie Pinel, p. 69-83.

${ }^{4}$ Voir, dans ce numéro, l'article d'Alexandre Giunta, p. 51-67.

${ }^{5}$ Voir, dans ce numéro, l'article d' Élodie Pinel., cité ci-dessus.

${ }^{6}$ Voir, dans ce numéro, l'article de Florian Besson, p. 35-50.

${ }^{7}$ Voir, dans ce numéro, l'article de Stéphanie Huart, p. 85-101.

${ }^{8}$ Voir, dans ce numéro, l'article de Florian Besson, cité ci-dessus
} 
l'articulation entre les diverses communautés et la construction d'un terrain commun de rites, de coutumes et de relations codifiées, révélant ainsi la pluralité des groupes qui se dessine en Orient et réduisant la part du fait religieux dans leur définition.

Mais ces réalités peuvent également revêtir une forme institutionnelle, organisée, établie à partir de chartes et de privilèges. Ainsi Alexandre Giunta ${ }^{9}$ envisage-t-il la construction d'une communauté à la fois urbaine et ethnique dans le cadre de la péninsule ibérique : celle des Francos, dont il retrace l'institutionnalisation et le processus d'ancrage dans le tissu urbain. La fondation d'une ville pour accueillir une communauté, le regroupement en quartiers ethniques, la délimitation d'activités économiques réservées aux étrangers, sont autant d'expressions de l'émergence et de la consolidation d'une communauté. En analysant les groupes et les réseaux interpersonnels résultant de ce processus ainsi que les phénomènes d'assimilation, Alexandre Giunta analyse la constitution et la dissolution de la communauté, suivant ainsi le processus du début à la fin.

Toutefois, au-delà de leur composante ethnique et de leur existence institutionnelle, les communautés urbaines médiévales reposaient sur divers éléments unificateurs, plus ou moins spontanés. C'est ce qu'aborde Cléo $\operatorname{Rager}^{10}$ dans le cas de Troyes au $X V^{\mathrm{e}}$ siècle. Elle y présente une communauté urbaine unie autour de la fidélité royale retrouvée, à travers un épisode de 1429 où Charles VII prend le contrôle de la ville. La communauté est alors consolidée «d'en-haut», autour de la personne du roi, à travers une politique de communication dirigée à l'attention de la communauté urbaine et entreprise par les officiers royaux (ostentation de signes d'appartenance, courriers lus par les crieurs de la ville...).

\footnotetext{
${ }^{9}$ Voir, dans ce numéro, l'article d'Alexandre Giunta, cité ci-dessus.

${ }^{10}$ Voir, dans ce numéro, l'article de Cléo Rager, cité ci-dessus. 
L'analyse du rôle joué par le facteur politique dans la redéfinition d'une communauté urbaine et de la construction d'une fidélité royale, conduit à interroger le ciment d'une communauté médiévale.

C'est également cette question que soulève Catherine Kikuchi ${ }^{11}$, en s'attachant cette fois à l'échelle intra-urbaine, à travers le cas d'une communauté de métiers: les imprimeurs et libraires de Venise. Parce qu'il n'existait pas de corporation avant le XVI siècle, cette étude interroge l'existence d'une communauté d'intérêts au sein de ce groupe, ainsi que l'organisation interne et les rapports entretenus avec les pouvoirs publics, alors même qu'il n'y a pas d'identité collective ou de communauté de métier institutionnellement reconnue.

Diane Chamboduc de Saint-Pulgent ${ }^{12}$ se propose quant à elle de repenser la communauté politique italienne, à partir de l'exemple de la ville de Lucques et de ses associations de quartier. L'attention portée aux milieux populaires permet ainsi de sortir du cadre institutionnel et d'interroger la question du sentiment d'appartenance des habitants à leur cité, sentiment qui transparaît à l'occasion des diverses manifestations publiques, telles que les fêtes, mais aussi les délibérations collectives.

Enfin, « faire communauté » appelle, en creux, la problématique de l'exclusion du groupe. À partir de quand est-on perçu comme un étranger à la communauté ? Quelles en sont les conséquences ? Ces questionnements, déjà présents dans l'article d'Alexandre Giunta ${ }^{13}$, sont abordés par Élodie Pinel ${ }^{14}$ d'un point de vue littéraire, à partir de son analyse du Mirouer des simples ames de Marguerite Porete. L'œuvre amène à interroger la définition de l'hérésie et de l'exclusion de la

\footnotetext{
${ }^{11}$ Voir, dans ce numéro, l'article de Catherine Kikuchi, cité ci-dessus.

12 Voir, dans ce numéro, l'article de Diane Chamboduc de Saint-Pulgent, cité cidessus.

${ }^{13}$ Voir, dans ce numéro, l'article d'Alexandre Giunta, cité ci-dessus.

${ }^{14}$ Voir, dans ce numéro, l'article d' Élodie Pinel., cité ci-dessus.
} 
communauté des chrétiens : à partir de quel moment un écrit religieux estil perçu comme hérétique ? Dans quelles formes littéraires s'inscrit-il ? Élodie Pinel aborde ici la dialectique entre le contenu d'un texte, qui se revendique comme un ferment de la communauté chrétienne, et sa réception, qui le rejette dans l'exclusion et la condamnation.

Mais cette exclusion de la communauté est aussi un instrument, voire une arme. Aussi Stéphanie Huart ${ }^{15}$ s'intéresse-t-elle aux modalités du bannissement autant qu'à ses enjeux, à travers le cas de la communauté urbaine de Valenciennes au XIV ${ }^{\mathrm{e}}$ siècle. Exclure, c'est aussi souder la communauté et assurer la continuité politique, de même que le maintien de la paix.

Ainsi, nous espérons avoir mis en lumière, grâce à ces articles, la variété des formes et pratiques que recouvre l'usage du terme « communauté » à la fin du Moyen Âge. Toutefois, ce ne sont là que les prémisses d'une réflexion qui mériterait une étude de plus grande ampleur, notamment sur le plan chronologique. À ce titre, soulignons la tenue d'un séminaire sur les communautés entre le $\mathrm{V}^{\mathrm{e}}$ et le $\mathrm{XI}$ siècle, organisé par Geneviève Bührer-Thierry, François Bougard et Régine Le Jan au sein du laboratoire du CNRS de l'université de Paris 1 PanthéonSorbonne $^{16}$, le LAMOP, qui témoigne de l'actualité scientifique de la question.

\footnotetext{
${ }^{15}$ Voir, dans ce numéro, l'article de Stéphanie Huart, cité ci-dessus.

${ }^{16}$ Séminaire, Les communautés ( $\mathrm{V}^{\mathrm{e}}-\mathrm{XI}^{\mathrm{e}}$ siècles) : formes, pratiques, interactions (1). Communautés locales et spatialisation des communautés, Université de Paris 1 Panthéon-Sorbonne, LAMOP, Geneviève Bührer-Thierry, François Bougard et Régine Le Jan, jeudi 15-17h, Sorbonne.
} 